\title{
Investigação do reflexo vermelho em recém-nascidos e sua relação com fatores da história neonatal*
}

\author{
INVESTIGATION OF THE RELATED FACTORS BETWEEN NEWBORN HISTORY \\ AND RED REFLEX
}

\section{INVESTIGACIÓN DEL REFLEJO ROJO EN RECIÉN NACIDOS Y SU RELACIÓN CON FACTORES DE LAHISTORIANEONATAL}

\author{
Ingrid Martins Leite Lúcio', Maria Vera Lúcia Moreira Leitão Cardoso², \\ Paulo César de Almeida ${ }^{3}$
}

\footnotetext{
* Extraído da dissertação "Método de avaliação visual aplicado ao recémnascido". Programa de Pós-Graduação em Enfermagem do Departamento de Enfermagem da Faculdade de Farmácia, Odontologia e Enfermagem - FFOE da Universidade Federal do CearáUFC, 2004

1 Enfermeira. Mestre em Enfermagem. Membro do Projeto Saúde Ocular-UFC/ CNPq. Discente do Curso de Doutorado da Universidade Federal do Ceará. Bolsista CAPES. ingrid_lucio @yahoo.com.br

2 Enfermeira.

Professora Adjunta do Departamento de Enfermagem da Universidade Federal do Ceará. Doutora em Enfermagem. Coordenadora da área saúde ocular da criança do Projeto Saúde Ocular-UFC/ CNPq.

cardoso@ufc.br

3 Estatístico. Doutor em Saúde Pública. Professor Adjunto da Universidade Estadual do Ceará. pc_almeida@ zipmail.com.br
}

\section{RESUMO}

O teste do reflexo vermelhoé parte importante do exame ocular do recém-nascido. Buscou-se investigálo em recém-nascidos prematuros (RNPT) e sua relação com fatores da história neonatal. Estudo descritivo, exploratório, quantitativo, realizado numa maternidade pública, em FortalezaCE, em março, abril e maio de 2004, com 114 RNPTs. Para o registro dos dados, utilizou-se um formulário próprio e para a realização do exame, um oftalmoscópio direto. Considerou-se pertinente os seguintes achados da história neonatal: $68 \%$ dos RNPTs obtiveram Apgar inferior a sete no primeiro minuto; 112 utilizaram oxigenoterapia e 68 , fototerapia. Quanto ao reflexo vermelho, $13(11 \%)$ apresentaram alteração, com associação estatística significativa entre este e o Apgar no primeiro minuto de vida $(p=0,041)$. Os achados preocuparam, pois, dez dos 13 RNPTs, com alteração no reflexo vermelho, tinham retinopatia da prematuridade.

$\begin{array}{ll}\text { DESCRITORES } & \text { KEY WORDS } \\ \text { Saúde ocular. } & \text { Eye health. } \\ \text { Prematuro. } & \text { Infant, premature. } \\ \text { Cuidado do lactente. } & \text { Infant care. } \\ \text { Enfermagem neonatal. } & \text { Neonatal nursing. }\end{array}$

\section{ABSTRACT}

The red reflex test is an important part of the newborn's ocular exam. This study investigated the relationship between red reflex in premature newborns (PN) and factors of the newborn history. It's a descriptive, exploratory, quantitative study conducted in a public maternity in Fortaleza, State of Ceará, with 114 PN between the months of April and May of 2004. For data registration was used a questionnaire, and for the ocular exam a direct ophthalmoscope. Data showed the following findings: $68 \%$ of PN had an Apgar under seven during the first minute; 112 were submitted to oxygen therapy; and 68 to phototherapy. As for the red reflex, 13 (11\%) showed alterations, with significant statistical association between red reflex and the Apgar during the first minute of life $(p=0.041)$. The results cause concern because 10 of the 13 PN with red reflex showed retinopathy of prematurity.

Neonatal nursing.

\section{RESUMEN}

El examen del reflejo rojo es parte importante del examen ocular del recién nacido. Se lo investigó en recién nacidos prematuros (RNPT) y su relación con factores de la historia neonatal. Estudio descriptivo, cuantitativo realizado en una maternidad pública, en Fortaleza-CE, en marzo, abril y mayo de 2004 con 114 RNPT. Para el registro de los datos se utilizó un formulario propio y para realización del examen un oftalmoscopio directo. Se consideró pertinente los siguientes hallazgos de la historia neonatal: un $68 \%$ de los RNPT obtuvieron Apgar inferior a siete en el primer minuto; 112 utilizaron oxígeno y 68 fototerapia. Cuanto al reflejo rojo, 13 (un 11\%) presentaron alteración, con asociación estadística significativa entre éste y el Apgar en el primer minuto de vida ( $\mathrm{p}=$ 0,041). Los hallazgos han causado preocupación, ya que 10 de los 13 RNPT con alteración en el reflejo rojo tenían retinopatía de la edad prematura.

\section{DESCRIPTORES}

Salud ocular.

Prematuro.

Cuidado del lactante.

Enfermería neonatal. 


\section{INTRODUÇÃO}

A elaboração e a incorporação de programas voltados para ações no âmbito da saúde ocular, com intervenções eficazes, ainda não constituem realidade efetiva na maioria dos países em desenvolvimento, como no Brasil, e, quando existentes, são dirigidos com maior afinco à atenção primária de saúde. Além disso, outra questão preocupante é que o aparecimento de algumas alterações visuais, especialmente na infância, pode manter estreita relação com as condições socioeconômicas da população, como a xeroftalmia, alteração que acomete a córnea, consequiência da deficiência de vitamina $\mathrm{A}$, que responde por 350.000 casos do total de 1,5 milhões de crianças cegas no mundo(1).

A criança mostra-se mais vulnerável a apresentar distúrbios visuais, por ter um rápido crescimento e desenvolvimento do sistema visual nos primeiros anos de vida. Uma "deficiência visual", constatada durante este período, pode estar intimamente ligada a um atraso do desenvolvimento infantil, que pode evoluir com sérias conseqüências para a vida adulta ${ }^{(2-3)}$. Estas consequiências agravam-se de acordo com o período de consolidação do diagnóstico, logo, quanto mais precoce, maior a chance de preveni-la ou tratar os agravos.

Dados precisos acerca da incidência e prevalência da cegueira em crianças são de obtenção difícil, haja vista sua raridade e, portanto, se fazer necessário selecionar largas amostras para estudos de prevalência e estudos longitudinais. Estudos desta natureza, entretanto, revelam uma prevalência pequena de cegueira infantil, em torno de 0,2 a 0,3 por 1000 crianças em países desenvolvidos e de 1,0 a 1,5 por 1000 crianças em países em desenvolvimento ${ }^{(3-4)}$.

No Brasil, em especial na região Nordeste, são escassos os dados referentes às causas mais comuns de deficiências visuais na infância, embora mais da metade dos agravos à saúde ocular sejam prevenidos e adequadamente tratados com o conhecimento e a tecnologia de hoje na área. Por esta razão, a importância de programas voltados para a promoção e prevenção da saúde ocular ${ }^{(5)}$. A perda da capacidade visual compromete a qualidade de vida do indivíduo e, quando estabelecida na infância, compromete o desenvolvimento global, envolvendo aspectos sensoriais, psicológicos e motores interferindo sensivelmente na socialização da criança, quer na família, escola ou outro núcleo de integração.

Diante do exposto, são necessários o cuidado e a adoção de intervenções multiprofissionais dirigidas à prevenção de alterações visuais a partir do pré-natal e, por conseguinte, no período pós e neonatal, procurando investigar fatores congênitos, distúrbios neurológicos e infecções maternas, como a rubéola, a sífilis e a toxoplasmose, que podem comprometer o desenvolvimento funcional da visão, e prevenir fatores adquiridos ocasionados por algumas terapêuticas, como a oxigenoterapia, a fototerapia e transfusões sangüíneas ${ }^{(6-7)}$, geralmente necessárias à manutenção/recuperação do estado de saúde em recém-nascidos prematuros.

Nos países plenamente desenvolvidos, as alterações mais comuns em crianças são detectadas na infância precoce. No Reino Unido, o exame ocular é realizado rotineiramente nos recém-nascidos, visando a promover, o mais precoce possível, a adequada orientação terapêutica, o aconselhamento genético e outras condutas de suporte às doenças oculares $^{(8)}$. No Brasil, esforços têm sido somados visando ações sistemáticas dirigidas à saúde ocular desde o período neonatal no âmbito multiprofissional.

O recém-nascido prematuro integra um grupo considerado como "de risco" para alterações visuais, não apenas por sua imaturidade fisiológica, mas também pela possibilidade de complicações, uso de suportes terapêuticos, extensos períodos de internamento, risco para infecções, entre outros. A realização criteriosa de uma anamnese com enfoque nos fatores de risco materno e neonatal, além daqueles familiares, com indícios oftalmológicos deveria constituir uma rotina, assim como a adoção de um teste de triagem conhecido como teste do reflexo vermelho, importante para a deteç̧ão precoce de tumores, retinopatia da prematuridade e catarata congênita.

Este teste é rápido, simples e realizado com o auxílio de um oftalmoscópio direto, preferencialmente com o ambiente em penumbra cujo objetivo é avaliar a qualidade dos meios transparentes do olho (córnea, cristalino e vítreo), além de possibilitar algumas informações sobre a retina. Quando presentes, as opacificações no eixo visual passam a constituir obstáculo à passagem e a chegada da luz à retina para a formação de imagens.

No cuidado de enfermagem, a prática envolvendo a realização do teste do reflexo vermelho encontra-se em construção, tanto ao nível de docência como assistência, assim como a avaliação visual sistemática. $\mathrm{O}$ teste do reflexo vermelho tem sido objeto de estudos exploratórios intrínsecos ao Projeto Saúde Ocular - área da Saúde Ocular da Criança desde o primeiro semestre de 2003, quando o mesmo foi introduzido a um método de avaliação visual proposto e aplicado ao recém-nascido ${ }^{(9)}$. No segundo semestre de 2004, dando seguimento ao aprofundamento teórico e prático relacionado ao teste, foi proposto um projeto tendo-o como objeto de estudo, no cunho de iniciação científica, pelo Programa de Bolsas de Iniciação Científica - PIBIC, e encontra-se em fase de conclusão. 
Diante da relevância do estudo para o cuidado de enfermagem ao recém-nascido, no âmbito global e da saúde ocular, objetivou-se investigar o reflexo vermelho em recém-nascidos prematuros e sua relação com fatores da história neonatal.

\section{MÉTODO}

Estudo descritivo-exploratório, quantitativo desenvolvido em uma instituição pública federal - maternidade, referência para o Município de Fortaleza e para o Estado do Ceará. Os setores escolhidos foram: 1) a Unidade de Internação Neonatal - UIN, 2) a Enfermaria “mãe-canguru”, e 3) o Alojamento Conjunto-AC.

A população do estudo foi constituída por recém-nascidos prematuros - RNPT, ou seja, aquele com Idade Gestacional - IG ao nascimento inferior a 37 semanas, admitido nos referidos setores. Foram incluídos na amostra aqueles RN cuja IG ao nascimento foi inferior a 37 semanas, segundo a avaliação pelo Capurro somático, independentemente da classificação da prematuridade - limítrofe, moderada ou extrema ${ }^{(10)}$. Além desta condição, no momento do exame, o recém-nascido $(\mathrm{RN})$ deveria ter a idade corrigida de até 40 semanas. Os critérios de exclusão foram: IG ao nascimento maior do que 37 semanas, condições que expressassem instabilidade clínica e restrição de manuseio. A amostra calculada foi representativa, considerando a variável "alteração ocular ou suspeita de doença ocular", com uma prevalência $\mathrm{P}$ de $10 \%{ }^{(8)}$, um erro amostral de $5 \%$ e um nível de significância também de $5 \%$.

Os dados foram coletados nos meses de março, abril e maio de 2004 e como meio de coleta utilizou-se um formulário composto por três partes: 1) história materna e história neonatal, 2) exame ocular e 3) condutas de enfermagem, tendo como base o instrumento utilizado em um estudo anterior ${ }^{(11)}$. Seu conteúdo englobou peculiaridades da visão do $\mathrm{RN}$, aspectos relevantes do histórico e anamnese, exame físico do olho e testes específicos para o período neonatal, como o teste do reflexo vermelho ou teste de Bruckner, importante para a observação de opacidades em algumas estruturas internas do olho.

Como este aspecto constituía uma prática nova para a condutora do exame, uma enfermeira, planejou-se um treinamento com uma oftalmologista, adquiriu-se um oftalmoscópio direto para sua observação, pelo Projeto Saúde Ocular/UFC/CNPq e, fez-se um levantamento de literatura que contemplasse o manuseio do equipamento e a técnica para a condução do teste. Orientações também foram feitas pela oftalmologista, que colaborou comprometendose a avaliar os RN que necessitassem de encaminhamento visando exames mais especializados.

Os dados que comportaram a história materna e neonatal foram obtidos em consultas aos registros do prontuário do $\mathrm{RN}$ e nos casos em que porventura não se encontraram registrados foram feitas indagações à mãe e/ou responsável, que colaborou como informante.
Para a realização do teste do reflexo vermelho, que é parte integrante do exame ocular do recém-nascido, foi utilizado um oftalmoscópio binocular direto. O oftalmoscópio dispõe de um disco que contém uma série de lentes esféricas, cujo poder varia de $+1,0 \mathrm{D}$ a $+20,0 \mathrm{D}$ e $-1,0 \mathrm{D}$ a $-20,0 \mathrm{D}$. Inicia-se o exame, segurando o oftalmoscópio a $20 \mathrm{~cm}$ do paciente com o disco posicionado no zero. Com um dos olhos colocado no orifício do oftalmoscópio direciona-se o mesmo ao olhar do paciente; um feixe de luz é emitido pelo aparelho sobre o centro pupilar. Nesta condição, observa-se um clarão vermelho que corresponde ao reflexo vermelho do fundo do olho ${ }^{(12)}$.

Para contemplar o objetivo deste artigo foram analisados e discutidos dados obtidos da primeira parte do formulário, no que diz respeito aos dados da história neonatal, e da segunda, a qual tratou do exame ocular, particularizando o teste do reflexo vermelho. Os dados investigados foram processados com o auxílio do software SPSS versão 11.0 e com base na Estatística descritiva e apresentados em forma de tabelas. $\mathrm{O}$ teste de Fisher foi utilizado para a análise das tabelas $2 \mathrm{X} 2 \mathrm{e} \mathrm{o}$ de Fisher-Freeman-Halton para as tabelas rXc. Para os testes, adotou-se o nível de significância de 5\%. As discussões dos resultados foram respaldadas em literaturas que visaram à importância do cuidado com a visão do RN precocemente e à detecção de alterações visuais nesta fase do ciclo vital.

O estudo foi aprovado pelo Comitê de Ética em Pesquisa do Complexo Hospitalar da Universidade Federal do Ceará COMEPE (protocolo 221/03). OTermo de Consentimento Livre e Esclarecido foi assinado pela mãe e/ou responsável legal do $\mathrm{RN}$, respeitando-se os aspectos éticos conforme a Resolução 196/96 do Conselho Nacional de Saúde - Ministério da Saúde.

\section{RESULTADOS E DISCUSSÃO}

A prática da observação do reflexo vermelho ainda não é evidenciada como rotina na assistência neonatal pelos profissionais de saúde, assim como a investigação de fatores da história neonatal que podem manter relação com alterações visuais e o reflexo vermelho. Neste estudo, o teste do reflexo vermelho foi introduzido pela primeira vez no exame ocular do recém-nascido $(\mathrm{RN})$ realizado e observado por uma enfermeira que integra o Projeto Saúde Ocular. Os RN que apresentaram alteração deste reflexo, em total de 13 de uma amostra de 114 recém-nascidos prematuros (RNPT), foram avaliados posteriormente pelo oftalmologista.

O teste do reflexo vermelho é usado para rastrear anormalidades do fundo de olho (seguimento posterior) e opacidades no eixo visual, como a catarata e opacidades de córnea, e todas as crianças devem ser avaliadas nos primeiros dias de vida. $\mathrm{O}$ resultado deste teste pode ser considerado negativo ou normal, quando o reflexo em ambos os olhos são equivalentes na cor, intensidade e clareza e não existem opacidades ou pontos brancos em alguma área, em um ou em ambos os reflexos observados. O resultado positivo ou anormal caracteriza-se de maneira oposta ao que foi mencionado ${ }^{(13)}$. 
Os RN foram avaliados no leito, berço ou incubadora, em decúbito dorsal, com uma leve flexão da cabeça, e com o ambiente em penumbra, pois isto torna mais evidente a observação do reflexo vermelho, favorecendo a dilatação fisiológica da pupila. Percebeu-se uma variação da coloração do reflexo, ora pálido, ora com áreas semelhantes à teia de aranha, ora vermelho-alaranjado intenso. Em relação à intensidade, algumas vezes observou-se diferença entre os olhos. No entanto, a literatura aponta que em RNPT, o remanescente da túnica vasculosa lentis, freqüentemente, é visível anterior ou posterior ao cristalino, ou ainda em ambas as posições, sendo o restante geralmente absorvido com o tempo, semelhante a uma completa ou parcial "teia de aranha"(14).

O profissional enfermeiro ao direcionar sua perspectiva de cuidado para a promoção da saúde ocular do RN e a prevenção de alterações deve se questionar a respeito de alguns pontos como, se, na coleta de dados da história materna, foram investigadas infecções, como rubéola, toxoplasmose, doenças sexualmente transmissíveis, entre outras, e casos de catarata, glaucoma e retinopatia na família; ou se foram identificados fatores que colaboram para o parto e nascimento prematuros; como foi a avaliação inicial do RN ao nascimento, quanto ao Apgar, idade gestacional e peso; se alguma alteração nas estruturas oculares externas foi observada; se o RN necessitou de oxigênio; se a credeização foi realizada corretamente; se alguma reação foi identificada; e quais futuros cuidados de enfermagem seriam dirigidos ao $\mathrm{RN}$.

Procedeu-se à caracterização da amostra, em relação as variáveis do nascimento, para uma compreensão geral deste contexto, e posteriormente, a exploração de algumas destas variáveis com o resultado do teste do reflexo vermelho.

Constatou-se quanto ao tipo de parto o predomínio de nascimentos por cesarianas (71\%); 48\% dos RN eram do sexo masculino e $52 \%$ do feminino. Ao verificar o registro do Apgar no primeiro minuto de vida, observou-se que 65 RN obtiveram índice regular (58\%) e 12 (10\%) índice baixo o que traduz condição de hipoxia ao nascimento. Ao observar o registro do Apgar no quinto minuto, viuse uma melhora considerável, pois $92(82 \%)$ obtiveram um bom índice.

Destacou-se o Apgar, porque a avaliação da vitalidade do RN é realizada rotineiramente utilizando a escala de Virgínia Apgar, no primeiro e quinto minuto de vida. Caso atinjam baixos escores, menores do que 4, os RN se encontram deprimidos e recebem assistência específica, como oxigenação, reanimação entre outros ${ }^{(15)}$. Este baixo escore deve ser considerado, em virtude da condição de hipoxia presente, que pode ser de moderada a grave, haja vista outros fatores que apresentam relação com o desenvolvimento neurológico deficiente e, consequientemente, podem favorecer alterações visuais, portanto requerem o uso do oxigênio em modalidades distintas e por tempo variável, agravado ainda pelas constantes flutuações.
Dentre os 77 (68\%) RN que apresentaram Apgar menor do que 7 no primeiro minuto, 16 sofreram anoxia grave e 15 anoxia moderada ao nascimento. Anoxia significa falta de oxigênio, suprimento reduzido de oxigênio. Talvez, cerca de $20 \%$ dos RN experimentem algum grau de anoxia. Os efeitos em longo prazo são difíceis de determinar. A anoxia prolongada está freqüientemente associada a consequiências como paralisia cerebral e retardo mental, períodos mais breves, parecem ter pouco efeito prolongado ${ }^{(16)}$.

A avaliação da idade gestacional - IG é essencial para uma boa prática tanto obstétrica como neonatal. A escala utilizada pelos profissionais da Instituição para a estimativa da idade gestacional, geralmente, foi a de Capurro, cujo registro estava no prontuário. Dentre os RN, apenas 7\% foram avaliados como prematuros extremos, ou seja, com idade gestacional inferior a 30 semanas. A maioria 88 (78\%) foi de RNPT - moderados, que inclui os RNPT com IG entre 30 e $34{ }^{6 / 7}$ semanas e extremos, que compreendem aqueles com IG inferior a 30 semanas $^{(10)}$.

O risco para infecção foi encontrado em $44 \%$, dado obtido do registro de impressões médicas, referente as primeiras 24 horas de vida. O risco para infecção geralmente esteve relacionado a infecções maternas e ao nascimento, sendo por parto normal, ruptura de bolsa e/ou aspiração de mecônio, sofrida por 22 (19\%) dos RN.

Estes aspectos apresentados foram inerentes à avaliação inicial do RN, nas primeiras horas de vida, constante no prontuário e relevantes para o profissional que objetiva dirigir seu cuidado à promoção da saúde ocular. Constituem componentes da história neonatal, que exigirão uma série de terapêuticas e condutas, que podem implicar em cuidados com os olhos, a fim prevenir complicações.

Outro fator que mereceu enfoque foi o peso ao nascimento, cujos dados evidenciaram peso mínimo de 630 gramas e máximo de 3125 gramas, com média de 1830 gramas. A prevalência de baixo peso, a princípio, tanto pode estar relacionada com a prematuridade como com o Retardo de Crescimento Intra-Uterino. $\mathrm{O}$ peso ao nascer varia intensamente com a duração da gestação e crianças que nascem com baixo peso, ou seja, com peso inferior a 2500 gramas, não necessariamente são pequenas para idade gestacional (PIG) ${ }^{(17)}$. As características descritas condizem com a literatura. Conhecê-las e identificá-las se faz importante para o enfermeiro, que deve utilizá-las para ampliar o conhecimento sobre o seu cliente e assim planejar o cuidado.

Outro aspecto da história neonatal investigado foi a respeito da credeização, um cuidado de rotina, o qual foi realizado em 41 (36\%) RN. O método profilático de Credé utiliza o nitrato de prata a $1 \%$, uma gota em cada um dos olhos ao nascer e previne contra a conjuntivite neonatorum ${ }^{(15,18)}$. Para esta profilaxia também pode ser utilizado o argirol a $20 \%$, porém, salienta-se que não se pode nomeá-la de credeização, 
mas sim profilaxia contra oftalmia neonatal. A solução é instilada no saco conjuntival ao nascimento e em alguns bebês causa edema e vermelhidão considerável nas pálpebras. Estes sintomas são evidenciados entre o segundo e quinto dia de vida ${ }^{(14)}$.

Além da profilaxia para a oftalmia, outros dois fatores foram investigados na história neonatal, antes de adentrar no exame ocular: o uso de oxigênio e o emprego de fototerapia, cujos dados encontram-se apresentados na Tabela 1.

Tabela 1 - Distribuição do número de recém-nascidos prematuros segundo a terapêutica MEAC - Fortaleza, março - maio - 2004

\begin{tabular}{lcc}
\hline Terapêtica & N & $\%$ \\
\hline Oxigenoterapia (dia) $(\mathrm{n}=112)$ & 80 & 71 \\
1 a 10 & 24 & 21 \\
11 a 20 & 05 & 4 \\
21 a 30 & 02 & 2 \\
31 a 40 & 01 & 1 \\
$>41$ & & \\
Fototerapia (dia) $(\mathrm{n}=68)$ & 36 & 53 \\
1 a 5 & 26 & 38 \\
6 a 10 & 06 & 9 \\
10 a 15 & -
\end{tabular}

Fonte: Maternidade-Escola Assis Chateubriand - MEAC - Prontuário. $\mathbf{N}=114$ recém-nascidos .

Encontrou-se registro de uso de oxigênio em 112 dos 114 prontuários consultados, ao se investigar os fatores da história neonatal, predominando um tempo compreendido no intervalo de 1 a 10 dias em 80 registros (71\%), 24 (21\%) por um intervalo de 11 a 20 dias. Um RN fez uso de oxigênio por mais de 41 dias, e ainda se encontrava sob a necessidade de oxigênio, mesmo depois de concluída sua avaliação visual, e, provavelmente, corre um grande risco de sofrer iatrogenias, secundárias ao uso prolongado do oxigênio.
O uso do oxigênio mantém relação com a impressão do diagnóstico médico síndrome do desconforto respiratório ou doença da membrana hialina apresentado por $104 \mathrm{RN}$. Esta patologia é o distúrbio respiratório mais freqüente nos RNPT, acometendo cerca de 10 a $15 \%$ dos RN com peso abaixo de $2,5 \mathrm{~kg}$ ao nascimento e $30 \%$ daqueles que têm menos de 34 semanas, exigindo como uma das terapêuticas a aplicação cuidadosa de medidas de suporte geral, assistência respiratória e terapia de reposição de surfactante exógeno ${ }^{(19)}$. São características predominantes quando da análise das variáveis relacionadas às condições de nascimento e peso dos RN do estudo.

As modalidades de oxigenoterapia utilizadas englobaram o oxigênio circulante, o oxigênio por capacete (hood), o CPAP (nasal) - pressão positiva contínua nas vias aéreas e a ventilação mecânica, de maneira isolada e com variações (associações), dependendo da evolução clínica do RN. A maioria deles iniciou a terapêutica com a ventilação mecânica, passando ao CPAP nasal até o desmame pelo oxi-hood. Todas objetivam administrar oxigênio com finalidade terapêutica. A administração e monitorização devem ser cuidadosas, a fim de evitar a administração desnecessária ou em altas concentrações, que podem produzir efeitos tóxicos, inclusive ao sistema visual ${ }^{(19)}$.

Reportando-se ao uso de fototerapia, apenas 68 dos 114 RN a utilizaram, a maioria 36 (53\%) de 1 a 5 dias sendo o equipamento de fototerapia mais utilizado o bilispot. Em alguns deles, durante a coleta deste dado, foi possível observar a proteção ocular, que se encontrava adequada, protegendo bem os olhos. Quando não, apresentou-se pouco fixada aos olhos do RN, que ao movimentar-se no berço, favorecia a diminuição da aderência do micropore ao couro cabeludo, provocando "folgas da proteção ocular" para a incidência da luz, que pode ocasionar degeneração da retina ${ }^{(14,20)}$. Após a explanação dos fatores de história neonatal investigadas, buscou-se a correlação do resultado do teste do reflexo vermelho com algumas variáveis relativas ao recém-nascido, esboçadas na Tabela 2 .

Tabela 2 - Relação entre o resultado de teste do reflexo vermelho e os fatores relacionados à história neonatal MEAC - Fortaleza, março - maio - 2004

\begin{tabular}{|c|c|c|c|}
\hline Fatores relacionados & $\begin{array}{c}\text { Reflex } \\
\text { Sem alteração }\end{array}$ & $\begin{array}{l}\text { vermelho } \\
\text { Com alteração }\end{array}$ & $\mathbf{P}$ \\
\hline Apgar ( $1^{\circ}$ minuto $) \mathrm{n}=113$ ) & & & 0,041 \\
\hline$<4$ & 9 & 3 & \\
\hline $4-6$ & 56 & 9 & \\
\hline $7-10$ & 35 & 1 & \\
\hline 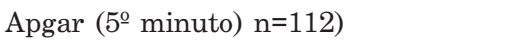 & & & 0,156 \\
\hline$<4$ & - & 1 & \\
\hline $4-6$ & 17 & 2 & \\
\hline $7-10$ & 82 & 10 & \\
\hline Uso de fototerapia (dia) $(n=68)$ & & & 0,744 \\
\hline 1 a 5 & 31 & 5 & \\
\hline 6 a 15 & 26 & 6 & \\
\hline Tempo de oxigenoterapia (dia) $(n=112)$ & & & 0,092 \\
\hline 1 a 15 & 87 & 9 & \\
\hline 16 a 45 & 12 & 4 & \\
\hline
\end{tabular}


Dos 114 recém-nascidos examinados, 13 apresentaram o reflexo vermelho alterado. Constatou-se que $12 \mathrm{RN}$ cujo reflexo vermelho apresentou-se alterado obtiveram Apgar no primeiro minuto inferior ao igual a seis; 6 fizeram uso de fototerapia de 6 a 15 dias; 9 fizeram uso de oxigênio de 1 a 15 dias e cinco de 16 a 45 dias. Verificou-se associação estatisticamente significativa entre reflexo vermelho e Apgar no primeiro minuto ( $\mathrm{p}=0,041)$; no entanto, não foi evidenciada uma relação significante entre reflexo vermelho e tempo de oxigenoterapia $(\mathrm{p}=0,092)$.

Alguns estudos, todavia, investigaram fatores que poderiam estar associados a uma patologia denominada retinopatia da prematuridade, que, quando presente, causa alteração no aspecto do reflexo vermelho, destacando a influência da oxigenoterapia ${ }^{(21)}$.

Os resultados deste estudo, cuja amostra foi composta por RN de muito baixo peso (peso $<1500 \mathrm{~g}$ ) e com idade gestacional inferior a 32 semanas, revelaram que a incidência de retinopatia da prematuridade - ROP foi inversamente proporcional ao peso e à idade gestacional. Também foi encontrada neste estudo associação estatisticamente significativa com o Apgar menor do que seis no primeiro e quinto minuto de vida e com o tipo de parto.

Mostrou também que RN que desenvolveram esta patologia fizeram uso de oxigênio de 28 a 30 dias, com predominância da ventilação mecânica, período superior ao daqueles que não a apresentaram. Outros fatores investigados no referido estudo que não apresentaram associação significativa com esta patologia foram $\mathrm{RN}$ com diagnóstico de síndrome do desconforto respiratório, RN que necessitaram de transfusão sanguínea, nutrição parenteral e fototerapia.

A retinopatia da prematuridade é uma doença que afeta a retina de crianças prematuras, principalmente aquelas com baixo peso. No prematuro, a retina encontra-se incompletamente vascularizada, sendo a isquemia o principal fator desencadeador de neovascularização da retina ${ }^{(22)}$.

Os treze recém-nascidos cujo reflexo vermelho estava alterado foram encaminhados para o oftalmologista, que posteriormente, ao realizar o exame de fundo de olho, constatou o diagnóstico de retinopatia da prematuridade, em diversos estádios. Foi possível acompanhar o exame de 10 destes, cujo reflexo estava alterado, a preparação para o exame com instilação de midriáticos e anestésicos, as condições do RN durante o procedimento do exame de fundo de olho e sua evolução após esta conduta realizada por uma oftalmologista.

\section{CONCLUSÕES}

O estudo mostrou-se relevante, pois apontou contribuições para o cuidado com a saúde global do recém-nascido, com enfoque na questão da saúde ocular, cujas ações ainda são pouco conhecidas e dirigidas de maneira sistemática do período neonatal. A investigação de fatores neonatais, rotineiramente, ocorre com vistas à detecção precoce de complicações que ponham em risco a saúde do recém-nascido e adequado tratamento, no entanto, poucos profissionais, tanto da ciência médica como da ciência da enfermagem, encontra-se preparados para investigar e acompanhar recém-nascidos que apresentem como complicação uma patologia ocular. Geralmente, como conduta, observa-se o encaminhamento destes bebês para serviços especializados, posteriormente a alta hospitalar, quando muitas outras condutas poderiam ser estabelecidas e executadas, envolvendo a equipe multiprofissional, mesmo durante o período de internação. Mudanças como esta, poderiam reduzir em longo prazo diagnósticos oftalmológicos tardios em crianças, que inclusive podem evoluir à cegueira, como estádios avançados de retinopatia da prematuridade e/ou glaucoma congênito.

Neste estudo foram evidenciados como fatores importantes quando se investigou a história neonatal com vistas à saúde ocular do recém-nascido a própria condição da prematuridade (100\% dos recém-nascidos), os baixos escores da escala de Apgar no $1^{\circ}$. minuto (58\% índice regular e $10 \%$ índice baixo), o baixo peso, com média de $1830 \mathrm{~g}$, o risco para infecção, registrado em $44 \%$ das impressões médicas, o uso de oxigênio $(71 \%$ dos recém-nascidos fizeram uso de 1 a 10 dias; $21 \%$ de 11 a 20 dias) e o emprego de fototerapia (53\% de 1 a 5 dias). A aplicação da profilaxia para a oftalmia neonatal, aplicada a 36\% dos recém-nascidos como uma de rotina, constitui uma eficaz medida para a redução dos casos de conjuntivite neonatal, eventualmente, observou-se caso de conjuntivite química.

Treze recém-nascidos apresentaram o reflexo vermelho alterado, posteriormente diagnosticados pelo oftalmologista diferentes graus de retinopatia da prematuridade. Houve associação estatisticamente significativa entre reflexo vermelho e Apgar no primeiro minuto de vida $(\mathrm{p}=0,041)$ e não significativa entre o reflexo vermelho e o tempo de oxigenoterapia $(\mathrm{p}=0,092)$. Ressalta-se que estas associações foram traçadas de maneira exploratória e merecem estudos posteriores para aprofundamento teórico, assim como a realização do teste do reflexo vermelho.

Acredita-se que o estudo aportará significativa contribuição para delinear estratégias de triagem visual no período neonatal, com a inserção do enfermeiro, dirigidas não somente a $\mathrm{RN}$ prematuros, mas também a todos, que podem ser expostos a outros fatores, que não a prematuridade. 


\section{REFERÊNCIAS}

1. Whitcher JP, Srinivasan M, Upadyay MP. Corneal blindness: a global perspective. Bull World Health Org. 2001;79(3):214-21.

2. Foster A, Gilbert C. Epidemiology of childness blindness. Eye. 1992;(6):173-6.

3. Bischh F. Análisis epidemiológico de la ceguera. Arch Chil Oftalmol. 1995;52(1):55-70.

4. Foster A. Curso de epidemiologia de causas de cegueira. Bucaramanga: Fundación Oftalmologica de Santander; 1996.

5. Albuquerque CR, Alves JGB. Afecções oculares em crianças de baixa renda atendidas em um oftalmológico na cidade de Recife PE. Arq Bras Oftalmol. 2003;66(6):831-4.

6. Hugonnier-Clayette S, Magnard P, Bourron-Madignier M, Hullo A. As deficiências visuais na infância: deficiências e adaptações. São Paulo: Manole; 1982. p. 7-22.

7. Nettina SM. Prática de enfermagem. $6^{\mathrm{a}}$ ed. Rio de Janeiro: Guanabara Koogan; 1998. v. 3.

8. Endriss D, Ventura LMVO, Diniz JR, Celino AC, Toscano J. Doenças oculares em neonatos. Arq Bras Oftalmol. 2002;65(5):551-5.

9. Lúcio IML. Método de avaliação visual aplicado ao recém-nascido [dissertação]. Fortaleza: Departamento de Enfermagem, Universidade Federal do Ceará; 2004.

10. Egewarth C, Pires FDA, Guardiola A. Avaliação da idade gestacional de recém-nascidos pré-termo através do exame neurológico e das escalas neonatais e obstétricas. Arq Neuropsiquiatr. 2002;60(3B):755-9.

11. Lúcio IML. Percepção da mãe co-participante da estimulação visual: uma estratégia de cuidado aplicada ao recém-nascido de baixo-peso [monografia]. Fortaleza: Departamento de Enfermagem, Universidade Federal do Ceará; 2002.
12. Freitas JAH. Oftalmologia básica. Rio de Janeiro: Colina; 1990.

13. American Academy of Pediatrics. Section Ophthalmology. Red reflex examination in infants. Pediatrics. 2002;109(5):980-1.

14. Fredrick DR. Assuntos especiais de interesse pediátricos. In: Voughan D, Asbury T, Riordan-Eva P. Oftalmologia geral. $4^{\mathrm{a}}$ ed. São Paulo: Atheneu; 1997. p. 340-7.

15. Schmitz EM. A enfermagem em pediatria e puericultura. São Paulo: Atheneu; 2000. p. 145-220.

16. Bee H. A criança em desenvolvimento. $7^{\text {a }}$ ed. Porto Alegre: Artes Médicas; 1996.

17. Kilsztajn S, Rossbach A, Carmo MSN, Sugahara GTL. Assistência pré - natal, baixo peso e prematuridade no Estado de São Paulo, 2000. Rev Saúde Pública. 2003;37(3):303-17.

18. Sociedade de Pediatria de São Paulo. Departamento de Neonatologia. Manual de neonatologia. $2^{\mathrm{a}}$ ed. rev. ampl. atual. Rio de Janeiro: Revinter; 2000.

19. Tamez RN, Silva MJP. Enfermagem na UTI neonatal: assistência ao recém-nascido de alto risco. Rio de Janeiro: Guanabara Koogan; 1999.

20. Naganuma M, Barbosa K, Fogliano I. Procedimentos técnicos de enfermagem em UTI neonatal. São Paulo: Atheneu; 1995.

21. Bancalari MA, González RCVC, Pradenas KI. Retinopatía del prematuro: incidencia y factores asociados. Rev Chil Pediatr. 2000;71(2):114-21

22. Liarth JCS, Meneses ES, Gonçalves JOR, Gonçalves EA, Aguiar AM. Retinopatia da prematuridade - estudo epidemiológico de 348 pacientes. RASPP Rev Assoc Saúde Pública Piauí. 1999;2(1):44-7. 